\title{
Long-term evolution of Landau damping in the presence of transverse noise, feedback, and detuning
}

\author{
Sondre Vik Furuseth $\oplus^{*}$ \\ CERN, 1211 Geneva, Switzerland, and EPFL, 1015 Lausanne, Switzerland \\ Xavier Buffat \\ CERN, 1211 Geneva, Switzerland
}

(Received 28 November 2019; accepted 18 February 2020; published 4 March 2020)

\begin{abstract}
The effect of Landau damping is often calculated assuming a Gaussian beam distribution in all transverse degrees of freedom, which agrees reasonably well with beam measurements. However, the stability of the beam is strongly dependent on the details of the distribution. The present study focuses on the slow evolution of the transverse bunch distribution, for a bunch excited by a coherent white noise source, damped by a transverse feedback system, and with detuning dependent on the transverse actions. The mechanism is modeled by the Fokker-Planck equation. It corresponds to a diffusion that is zero for particles with tune equal to the average tune of the bunch, and which is growing quadratically with the tune in the vicinity. The evolving distributions are then used to calculate the evolving stability diagrams, and thus the long-term evolution of the Landau damping. The relative effective octupole current is reduced faster for stronger noise, stronger linear detuning coefficients and weaker damper gain. The relevant parameters for this mechanism are scanned. With relevant parameter values for the LHC, this mechanism can cause a reduction of the effective octupole current by at most $10 \% / \mathrm{h}$.
\end{abstract}

DOI: 10.1103/PhysRevAccelBeams.23.034401

\section{INTRODUCTION}

In synchrotrons, the beam is kept stable partially by Landau damping due to the tune spread within each bunch. The stability diagram [1] in plane $j \in\{x, y\}$ can be calculated as

$\frac{1}{\Delta Q_{\mathrm{coh}, j}}=-\int_{0}^{\infty} \mathrm{d} J_{x} \int_{0}^{\infty} \mathrm{d} J_{y} \frac{J_{j} \frac{\partial \Psi\left(J_{x}, J_{y}\right)}{\partial J_{j}}}{Q-Q_{\mathrm{j}}\left(J_{x}, J_{y}\right)+i \epsilon}$,

and corresponds to the stability limit for the complex tunes $\Delta Q_{\mathrm{coh}, j}$ of the coherent modes. Because the stability diagram does not depend on the equilibrium distribution $\Psi$, but on the derivative of it, the stability can be changed significantly by a small change in the distribution. The stability diagram can be derived from the linearized Vlasov equation. Within this approximation, the transverse equilibrium distribution is typically considered constant [2]. The stability diagram of different equilibrium distributions have been calculated in the past [3-6]. The effect of longitudinal rf noise on the beam

\footnotetext{
*sondre.vik.furuseth@cern.ch
}

Published by the American Physical Society under the terms of the Creative Commons Attribution 4.0 International license. Further distribution of this work must maintain attribution to the author(s) and the published article's title, journal citation, and DOI. losses has also been studied [7,8], while transverse noise and diffusion has been given less attention, especially with a focus on loss of stability.

In a recent experiment in the LHC, transverse Landau damping was lost due to transverse noise driven diffusion $[9,10]$. Such an instability cannot be explained by the linearized Vlasov equation. It is expected that an accurate description of this mechanism involves the presence of both noise and wakefields [3]. Here, we consider an analytical theory explaining how the transverse equilibrium distribution changes slowly in the presence of transverse coherent noise, due to the combined effects of a transverse tune spread and a transverse feedback. The higher-order slow change of the distribution due to the wakefields themselves has at this stage been neglected, but may be required to explain the observed loss of Landau damping. A theory for a single transverse plane (1D), which has already been published [11], will here be extended to both transverse planes (2D). The stability of the slowly evolving beam, under the influence of wakefields, is at each time step evaluated by the linearized Vlasov equation through the stability diagram. The ultimate goal is to have an analytical model that predicts how fast the stability threshold is approached, to guide the search for optimal machine and beam parameters, mitigating this mechanism and maximizing the latency, relevant for HL-LHC and other future projects. 


\section{THEORY}

The calculation consists of 4 steps: Sec. II A Derive an expression for the change of the action for each particle after a kick, taking into account both the tune spread and the transverse feedback; Sec. II B Consider the change of action as a Wiener process with a drift, and derive the Fokker-Planck equation for the particle density distribution of the bunch; Sec. II C Solve the Fokker-Planck equation to get the time evolution of the distribution, both analytically and numerically; Sec. II D Calculate the evolving stability diagram numerically with PySSD [12], as the distribution evolves with time.

The 4-step calculation may be applied to various sources of tune spread. Here we shall discuss the case when the tune spread is achieved with Landau octupoles (II E).

\section{A. Transverse feedback and decoherence}

We will consider particles of a bunch in a circular accelerator, using for either transverse plane normalized canonical coordinates for the particles [13]

$$
\begin{aligned}
& x=\frac{X}{\sqrt{\beta \varepsilon_{0}}}=\sqrt{2 J} \cos (\phi), \\
& p=-\frac{1}{\sqrt{\beta \varepsilon_{0}}}\left(\alpha X+\beta \frac{\mathrm{d} X}{\mathrm{~d} s}\right)=-\sqrt{2 J} \sin (\phi),
\end{aligned}
$$

where $X$ is the offset from the design orbit, $s$ is the position in the beamline, $\alpha$ and $\beta$ are Twiss parameters, $\varepsilon_{0}$ is the initial beam emittance and $\phi$ is the canonical conjugate of $J$, which is the normalized absolute particle action, given in units of $\varepsilon_{0}$. It follows that $J$ can be written as

$$
J=\frac{1}{2}\left(x^{2}+p^{2}\right)
$$

and the emittance is given relative to the bunch centroid

$$
\frac{\varepsilon}{\varepsilon_{0}}=\frac{1}{2}\left\langle(x-\langle x\rangle)^{2}+(p-\langle p\rangle)^{2}\right\rangle,
$$

where the angle brackets signify the average over the bunch distribution.

If a bunch is kicked away from the design orbit by $\Delta p=k$, the action of a particle is changed to

$$
J_{k}=J_{0}-k \sqrt{2 J_{0}} \sin \left(\phi_{0}\right)+\frac{1}{2} k^{2},
$$

where $J_{0}$ and $\phi_{0}$ are the action and phase of the particle prior to the kick. Assuming a uniform phase distribution per action, the average action growth per initial action is

$$
\left\langle\Delta J\left(k, J_{0}\right)\right\rangle_{\phi_{0}}=\frac{1}{2} k^{2},
$$

independent of the initial action. If the bunch decoheres completely, shifting the centroid to the origin, it is trivial to show that the subsequent emittance growth is

$$
\frac{\Delta \varepsilon}{\varepsilon_{0}}=\frac{k^{2}}{2},
$$

where $\varepsilon_{0}$ was the emittance before the kick. This process requires that the individual particles have an incoherent tune offset $\Delta Q$ relative to the tune $Q_{c}$ of the bunch centroid. On the other hand, if there is no tune spread, but instead a transverse feedback that over multiple turns can push the bunch back to its design orbit with a gain $g$, the emittance growth, and correspondingly the action change, will be reduced.

The goal of this paper is to understand the change in the distribution after a kick, when there is both a transverse tune spread and a transverse feedback. There already exists an expression for the subsequent emittance growth in such a configuration [14]. We will take a similar approach, which was detailed in [11]. In contrast to previous research, we are not interested in the average change, but rather the change of action of each individual particle, which in this formalism is given by

$$
\begin{aligned}
\Delta J= & \frac{k^{2}}{2} \frac{\left(1-\frac{g}{2}\right)^{2} 4 \pi^{2} \Delta Q^{2}}{\left(\frac{g}{2}\right)^{2}+\left(1-\frac{g}{2}\right) 4 \pi^{2} \Delta Q^{2}}+k \sqrt{2 J_{0}}\left(1-\frac{g}{2}\right) \\
& \times \frac{\cos \left(\phi_{0}\right)\left(\frac{g}{2}\right) 2 \pi \Delta Q-\sin \left(\phi_{0}\right)\left(1-\frac{g}{4}\right) 4 \pi^{2} \Delta Q^{2}}{\left(\frac{g}{2}\right)^{2}+\left(1-\frac{g}{2}\right) 4 \pi^{2} \Delta Q^{2}} \\
= & \frac{1}{2} k^{2} L^{2}+k \sqrt{2 J_{0}}\left[M \cos \left(\phi_{0}\right)+N \sin \left(\phi_{0}\right)\right] \\
= & \frac{1}{2} k^{2} L^{2}+k \sqrt{2 J_{0}} \sqrt{M^{2}+N^{2}} \cos \left(\phi_{0}-\operatorname{atan}\left(\frac{M}{N}\right)\right),
\end{aligned}
$$

where $L, M$, and $N$ are functions of $\Delta Q$ and $g$. The step-bystep derivation is given in the Appendix A. The first term of Eq. (8) is an average growth, while the second term is a spread based on the phase of the particle. By averaging over the distribution, one gets the same expression for the emittance growth as in [14], assuming a uniform phase distribution. Equation (8) simplifies to Eq. (5) in the limit $g \ll \Delta Q$, and to 0 in the limit $g \gg \Delta Q$.

The expression in Eq. (8) has been derived under the assumption that the reduction of the transverse offset comes from the transverse feedback, not the decoherence $(g \gg \Delta Q)$. Furthermore,

$$
M^{2}+N^{2}=L^{2}+\frac{\left(1-\frac{g}{2}\right)^{2}\left(\frac{g}{4}\right)^{2}(2 \pi \Delta Q)^{4}}{\left[\left(\frac{g}{2}\right)^{2}+\left(1-\frac{g}{2}\right)(2 \pi \Delta Q)^{2}\right]^{2}},
$$

where the second term on the right-hand side is negligible for all relevant values of $\Delta Q$ and $g$. Hence, one can rewrite Eq. (8) as 


$$
\Delta J=\frac{1}{2} k^{2} L^{2}+k \sqrt{2 J_{0}} L \cos \left(\tilde{\phi}_{0}\right),
$$

where $\tilde{\phi}_{0}=\phi_{0}-\operatorname{atan}\left(\frac{M}{N}\right)$, which will still be uniformly distributed for each $J_{0}$, and

$$
L^{2}(g, \Delta Q)=\frac{\left(1-\frac{g}{2}\right)^{2} 4 \pi^{2} \Delta Q^{2}}{\left(\frac{g}{2}\right)^{2}+\left(1-\frac{g}{2}\right) 4 \pi^{2} \Delta Q^{2}},
$$

with a maximum value of $\left(1-\frac{g}{2}\right) \approx 1$.

In the derivation of Eq. (10), the incoherent tune offset $\Delta Q$ was assumed constant. However, in general it depends on $J$, which changes during this process. Right after the kick, the action is changed to $J_{k}$, given by Eq. (5). Due to the feedback, the action will be reduced back toward $J_{0}$. The variation of action during the process will be taken into account by calculating the average of Eq. (10) from $J_{0}$ to $J_{k}$ in the following manner

$$
\begin{aligned}
\overline{\Delta J} & =\frac{1}{J_{k}-J_{0}} \int_{J_{0}}^{J_{k}} \Delta J\left(J_{0}\right)+\left.\left(J^{\prime}-J_{0}\right) \frac{\partial \Delta J}{\partial J}\right|_{J=J_{0}} \mathrm{~d} J^{\prime} \\
& =\Delta J\left(J_{0}\right)+\left.\frac{J_{k}-J_{0}}{2} \frac{\partial \Delta J}{\partial J}\right|_{J=J_{0}} \\
& =\Delta J\left(J_{0}\right)+\frac{1-\frac{g}{4}}{1-\frac{g}{2}} \cdot k^{2} \sin ^{2}\left(\phi_{0}\right) J_{0} \frac{\partial L^{2}}{\partial J}+\mathcal{O}\left(k^{4}\right) .
\end{aligned}
$$

Note that the only impact of the variation of $J$ after the kick is through the action dependence of $\Delta Q(J)$. The values of $J_{0}$ and $\phi_{0}$ are supposed to be taken prior to the kick. Terms that will become zero when averaging over $\phi_{0}$ were dropped.

\section{B. Fokker-Planck equation in action}

In Sec. II A we derived Eq. (12), an expression for the change of action after a kick $k$, and how it depends on the action-phase coordinates prior to the kick, $\overline{\Delta J}\left(k, J_{0}, \phi_{0}, \Delta Q_{0}, g\right)$, from here on referred to as $\Delta$. Next, we have to derive how this change of action will change the distribution $\Psi$. To do so, we write the master equation $[15,16]$, a convolution of the distribution before the change $\Delta$ with the probability distribution of the change

$\Psi(J, t+\tau)=\int_{-\infty}^{\infty} \Psi(J-\Delta, t) \varphi(\Delta ; J-\Delta, \Psi) \mathrm{d} \Delta$.

The particle distribution is assumed uniformly distributed in $\phi$. The dependence of $\Delta$ on $\phi$ has been taken into account in the probability distribution $\varphi(\Delta)$. By doing so, the two-dimensional problem has become a one-dimensional one. All terms on the right-hand side are evaluated at time $t$.

When one kick $k$ becomes a coherent white noise source, the change of action can be considered a stochastic process, described by the Fokker-Planck equation [17]

$$
\partial_{t} \Psi=-\partial_{J}(U \Psi)+\partial_{J}^{2}(J D \Psi),
$$

with drift and diffusion coefficients

$$
\begin{gathered}
U(J, \Psi)=\int_{-\infty}^{\infty} \frac{\Delta}{\tau} \varphi(\Delta ; J, \Psi) \mathrm{d} \Delta, \\
D(J, \Psi)=\frac{1}{J} \int_{-\infty}^{\infty} \frac{\Delta^{2}}{2 \tau} \varphi(\Delta ; J, \Psi) \mathrm{d} \Delta,
\end{gathered}
$$

where $\tau$ is the time interval between each kick. The normalization of $D$ by $J$ will be convenient in the following. Equation (14) follows directly from a Taylor expansion of Eq. (13). See a short derivation in Appendix B.

The probability distribution for the change of action after a kick, derived from Eq. (10), can be written as

$$
\varphi(\Delta, J, \Psi)=\frac{F(k) \mathrm{d} k}{\pi \sqrt{2 J k^{2} L^{2}-\left(\Delta-\frac{k^{2}}{2} L^{2}\right)^{2}}},
$$

and it is defined only between the singularities. $F(k)$ is the probability distribution of the kicks, assumed to have zero mean and standard deviation $\sigma_{k}$.

The next step is to calculate the coefficients $U(J, \Psi)$ and $D(J, \Psi)$. The integral over $\Delta$ must be done first, as its limits depend on $k$. The coefficients become

$$
\begin{gathered}
D(J, \Psi)=\frac{\sigma_{k}^{2}}{2 \tau} \cdot L^{2}, \\
U(J, \Psi)=D+\frac{1-\frac{g}{4}}{1-\frac{g}{2}} J \frac{\mathrm{d} D}{\mathrm{~d} J} .
\end{gathered}
$$

The second term of $U$ comes from the inclusion of dynamically evolving $\Delta Q$ during the decoherence process.

The last step is to insert the expressions for $U$ and $D$ in Eq. (17) into the Fokker-Planck equation in Eq. (14). The first term of $U$ cancels $\partial_{J}\left[\partial_{J}(J) D \psi\right]$. In the assumed limit of small $g$, the second term cancels the term $\partial_{J}\left[J \partial_{J}(D) \psi\right]$. Thus, the Fokker-Planck equation turns into the wellknown diffusion equation

$$
\partial_{t} \Psi=\partial_{J}\left[J D \partial_{J}(\Psi)\right] .
$$

This equation could also be obtained by assuming a time reversal symmetry on the microscopic level. That is, the probability of going from $J_{a}$ to $J_{b}$ is equal to the process of going back, or $\varphi\left(J_{b}-J_{a} ; J_{a}\right)=\varphi\left(J_{a}-J_{b} ; J_{b}\right)$.

If we would have assumed constant tunes during the feedback and decoherence process of a single kick, the $D$ in Eq. (18) would have been inside the inner derivative. That partial differential equation (PDE) would have corresponded to a stochastic process where the probabilities of reducing and increasing the action by $\Delta$ from an initial 
action $J_{0}$ were equal, or $\varphi\left(\bar{\Delta}+\Delta ; J_{0}\right)=\varphi\left(\bar{\Delta}-\Delta ; J_{0}\right)$, where $\bar{\Delta}=k^{2} L^{2} / 2$ is assumed small. Such a process can easily be modeled with a macroparticle simulation, and an excellent agreement was found in [11]. However, it is not an accurate model of real beam dynamics, and will not be investigated further. It is a subtle, but important distinction from the process modeled by Eq. (18).

\section{Extension to $2 D$ action space}

We will now extend the theory from a single transverse plane to both transverse planes. The transverse detuning in high energy synchrotrons is usually generated by octupole magnets in a controlled way so that it depends on both transverse actions [1]. The expression for the linear detuning will be discussed in Sec. II E.

We want to go from Eq. (14) to a general 2D FokkerPlanck equation like

$\partial_{t} \Psi=\sum_{j=0}^{1} \frac{\partial}{\partial J_{j}}\left[-U_{j} \Psi+\sum_{i=0}^{1} \frac{\partial}{\partial J_{i}}\left(\sqrt{J_{j} J_{i}} D_{j i} \Psi\right)\right]$,

where the indexes $i$ and $j$ go over the two transverse planes, with definitions of $U_{j}$ and $D_{j i}$ as before. This equation can be obtained by doing a 2D Taylor expansion as in Appendix B. As for a single plane, we still have $U_{j} \propto$ $\sigma_{k j}^{2}+\mathcal{O}\left(\sigma_{k j}^{4}\right)$ and $D_{j j} \propto \sigma_{k j}^{2}+\mathcal{O}\left(\sigma_{k j}^{4}\right)$. Since the external noise in the two planes are considered uncorrelated, $\left\langle k_{x} k_{y}\right\rangle=0$, the coupling diffusion coefficients can be considered negligible, because $D_{x y}=D_{y x} \propto \sigma_{k x}^{2} \sigma_{k y}^{2} \ll D_{j j}$.

The Fokker-Planck equation in 2D transverse action space, which corresponds to Eq. (18) for one transverse plane, can therefore be written as

$$
\frac{\partial \Psi}{\partial t}=\frac{\partial}{\partial J_{x}}\left(J_{x} D_{x x} \frac{\partial \Psi}{\partial J_{x}}\right)+\frac{\partial}{\partial J_{y}}\left(J_{y} D_{y y} \frac{\partial \Psi}{\partial J_{y}}\right) .
$$

The diffusion coefficients are given as before,

$$
D_{j j}=\frac{\sigma_{k j}^{2}}{2 \tau} \cdot L^{2}\left[g_{j}, \Delta Q_{j}\left(J_{x}, J_{y}\right)\right],
$$

where we have explicitly written that $L^{2}$ depends on the detuning and feedback gain in the given transverse plane.

\section{Solving the Fokker-Planck equation}

In Sec. II B we studied the Fokker-Planck equation, and found that the problem at hand could be written as Eq. (18) in 1D and Eq. (20) in 2D. The next goal is to solve the Fokker-Planck equation, which will be done in three steps: (i) Change the amplitude variable to $r=\sqrt{2 J}$, interpreted as the radius in $(x, p)$-phase space; (ii) Find an analytical solution for two special cases; (iii) Write a numerical PDE solver for the general case.

\section{Change of variable}

The independent variables will be changed first of all in order to express the initial beam distribution with a familiar Gaussian, and second of all because a uniformly spaced grid in $r$ will better encompass the details of the change than a uniformly spaced grid in $J$. Therefore, the numerical solver that will be introduced in Sec. II C 3, will converge faster when using a grid in $r$ than in $J$.

The change of variables is achieved by using the chain rule, $\partial_{J} \cdot=\partial_{J}(r) \partial_{r} \cdot$, where $\partial_{J}(r)=1 / r$. Then, the two following expressions for the change in the distribution are equivalent

$$
\begin{aligned}
\partial_{t} \Psi & =\frac{\partial}{\partial J}\left[J D \frac{\partial \Psi}{\partial J}-U \Psi\right], \\
& =\frac{1}{r} \frac{\partial}{\partial r}\left[r \frac{D}{2} \frac{\partial \Psi}{\partial r}-U \Psi\right] .
\end{aligned}
$$

Note that the expressions for $D$ and $U$ have not been altered, as they are considered to be known functions.

\section{Analytical solution}

An analytical solution will be presented for the special case of $g=0$. In this case the Fokker-Planck equation takes the form

$$
\begin{aligned}
\partial_{t} \Psi & =\frac{D_{0}}{2} \frac{1}{r} \frac{\partial}{\partial r}\left[r \frac{\partial \Psi}{\partial r}\right], \\
D_{0} & =\frac{\sigma_{k}^{2}}{2 \tau} .
\end{aligned}
$$

This is the diffusion equation with a constant diffusivity $D_{0} / 2$. This is equal to what one expects from an incoherent noise source with the same variance $\sigma_{k}^{2}$ [18]. If the initial distribution in $\left(x, p_{x}\right)$ is a Gaussian with average action $\langle J\rangle(t=0)=1$, it will remain a Gaussian as

$$
\begin{aligned}
\Psi(t) & =\frac{1}{1+D_{0} t} \exp \left[-\frac{r^{2}}{2\left(1+D_{0} t\right)}\right] \\
& =\frac{1}{\langle J\rangle} \exp \left[-\frac{J}{\langle J\rangle}\right] .
\end{aligned}
$$

This thus corresponds to an emittance growth rate of $D_{0}$.

In another extreme limit, $g \gg \Delta Q$ and $\Delta Q \rightarrow 0$, the Fokker-Planck equation takes the form

$$
\partial_{t} \Psi=0
$$

The offsets from the kicks will be damped, without modifying the distribution, as expected.

\section{Numerical solution}

In the interesting regime, when both the feedback and the detuning will be relevant, the coefficients will depend on 


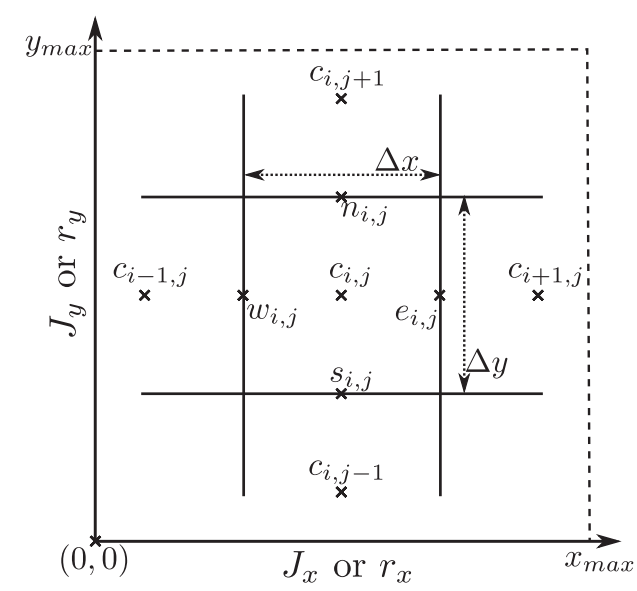

FIG. 1. Illustration of the FVM grid in PyRADISE. A uniform $N_{x} \times N_{y}$ cell grid covers the independent variables, either in the form of $J$ or $r$, from 0 to their respective maximal values. Each cell in the grid has a center $\left.c_{i, j}=\left[\left(i+\frac{1}{2}\right) \cdot \Delta x,\left(j+\frac{1}{2}\right) \cdot \Delta y\right)\right]$, and four edge points $e, w, n, s$, which are half a cell width or height in the respective compass directions. It follows naturally that $e_{i, j}=w_{i+1, j}$, etc.

the transverse actions, and we require a numerical solver to determine how the distribution will change. A PDE solver using the finite volume method (FVM) has been implemented and connected to a stability diagram calculator, which will be introduced in Sec. II D. The code will be referred to as PyRADISE (python radial diffusion and stability evolution).

The FVM has been applied because it ensures mass conservation in the interior region. The region of interest, and its discretization in a uniform $N_{x} \times N_{y}$ grid, is presented in Fig. 1. In the following, we assume that the solver uses the transverse actions as independent variables. The density in a certain cell, $\Psi_{i, j}$, is considered independent of $\left(J_{x}, J_{y}\right)$, and the distribution evolution comes from the diffusion and drift parameters at the four edge points, and density in the closest cell beyond each edge. At $J=0$, physics dictates that there must be a reflective boundary condition (BC), and at $J=J_{\max }$, one can choose between a homogeneous or inhomogeneous Neumann or Dirichlet BC. In the examples that follow, the $\mathrm{BC}$ at $J_{\max }=20$ is absorbing, representing an aperture in a real machine, the centroid tune, $Q_{c}$, is kept constant, and the grid is $700 \times 700$ in $2 \mathrm{D}$. The time integration is performed by scipy.integrate.solve_ivp [19], using a method based on a backward differentiation formula and a sparse Jacobian. The output is an array $\Psi(i, j, k)$, giving the density at the desired discrete times $t_{k}$, in all cells centered at $c_{i, j}$ in the 2D cell grid.

\section{Stability diagram}

The stability diagram must be calculated for each time $t_{k}$. Therefore, PySSD [12] is integrated in PyRADISE to numerically perform the integral in Eq. (1), using a uniform trapezoidal discretization in $\left(J_{x}, J_{y}\right)$, and thus calculate the stability diagrams for all distributions coming from the PDE-solver described in Sec. II C. Note from Eq. (1) that what we are interested in is the derivative of $\Psi$ with respect to the action. The distributions can therefore no longer be assumed to be piecewise constant, as in the FVM. First, the derivative with respect to $J_{x}$ is taken at the eastern (and western) edges as $\left(\Psi_{i+1, j}-\Psi_{i, j}\right) / \Delta x$, and equivalently for the derivative with respect to $J_{y}$ at the northern and southern edges. Then, the derivatives are linearly interpolated between the cell edges. Finally, the integral is performed numerically with $Q=\operatorname{Re} Q+\mathrm{i} \epsilon$, where $\epsilon \rightarrow 0$.

\section{E. Detuning by Landau octupoles}

The Landau octupoles are put in the LHC to produce a tune spread in order to keep the beam stable through Landau damping. The octupoles cause a tune spread in both transverse planes, relative to the average, which can be expressed as [1]

$$
\begin{aligned}
\Delta Q_{x / y} & =a_{x / y}\left(J_{x / y}-\left\langle J_{x / y}\right\rangle\right)+b_{x / y}\left(J_{y / x}-\left\langle J_{y / x}\right\rangle\right), \\
a_{x / y} & =520 \cdot I_{\mathrm{oct}} \varepsilon_{x / y, 0}, \\
b_{x / y} & =-364 \cdot I_{\mathrm{oct}} \varepsilon_{y / x, 0},
\end{aligned}
$$

where $J_{j}$ are the normalized actions, $a_{j}$ and $b_{j}$ are detuning coefficients that determine the tune spread amplitude [20], and $\varepsilon_{j, 0}$ are the initial transverse geometrical emittances. The factors 520 and -364 in Eq. (26) depend on the $\beta$-functions at the locations of the octupoles. The values can be increased by a factor $\in(2,4)$ by use of telescopic optics [21]. For a typical normalized emittance of $2.0 \mu \mathrm{m}$ and proton energy of $6.5 \mathrm{GeV}$, the geometrical emittance will be $\varepsilon_{x / y, 0}=289 \mathrm{pm}$. The maximum octupole current in the LHC is 550A. Thus, the maximum detuning coefficient is $a_{\max }=8.26 \times 10^{-5}$, according to Eq. (26). This expression for $\Delta Q$ should be inserted into the expression for $L^{2}$ in

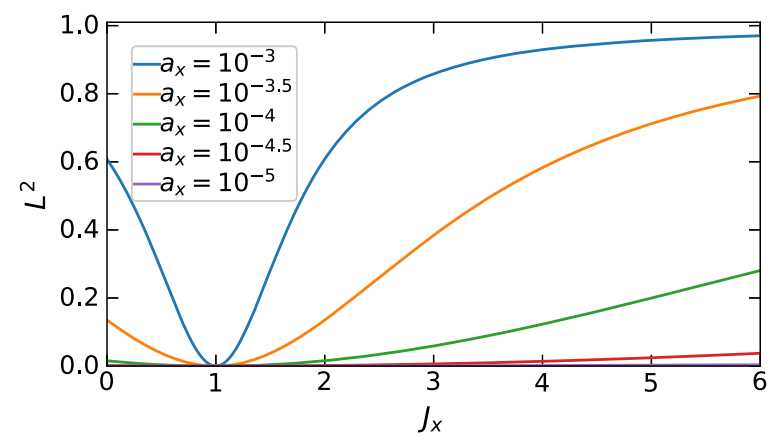

FIG. 2. Action dependence of $L^{2}$ for a horizontal damper gain $g_{x}=0.01$ and different values for the octupole detuning coefficient $a_{x}$, in the simplified case that $b_{x}=0$ [11]. 
Eq. (11). In a simplified model, when $b_{x}=0, L^{2}$ takes the shape illustrated in Fig. 2. We will focus our investigations on the diffusion caused by the combination of the active transverse feedback and detuning caused by Landau octupoles, because of its relevance for operation of the LHC.

\section{VERIFICATION}

\section{A. Change of action after a single kick}

First, we numerically evaluate the accuracy of Eq. (10). We have considered a 1D model with no dependence on the vertical plane, with $g_{x}=0.1, a_{x}=5 \times 10^{-4}$ and $b_{x}=0$. Simulations in this configuration have been run with $10^{7}$ macroparticles, for 150 turns, after a single horizontal kick of various amplitudes $k$ (in units of the beam size). The results of two numerical models are compared. The phase advance per particle has been kept constant in one set of simulations, and evolving dynamically as $J$ changes in another. The first abides by the assumptions used to derive Eq. (10), while the second is closer to the actual beam dynamics, used to derive Eq. (12).

The dependence of $\langle\Delta J\rangle$ and $\left\langle\Delta J^{2}\right\rangle$ on $J_{0}$ is presented in Fig. 3. They are compared to the theory given by Eq. (10) and modified theory including dynamical tunes given by Eq. (12). The modification did not change the expected
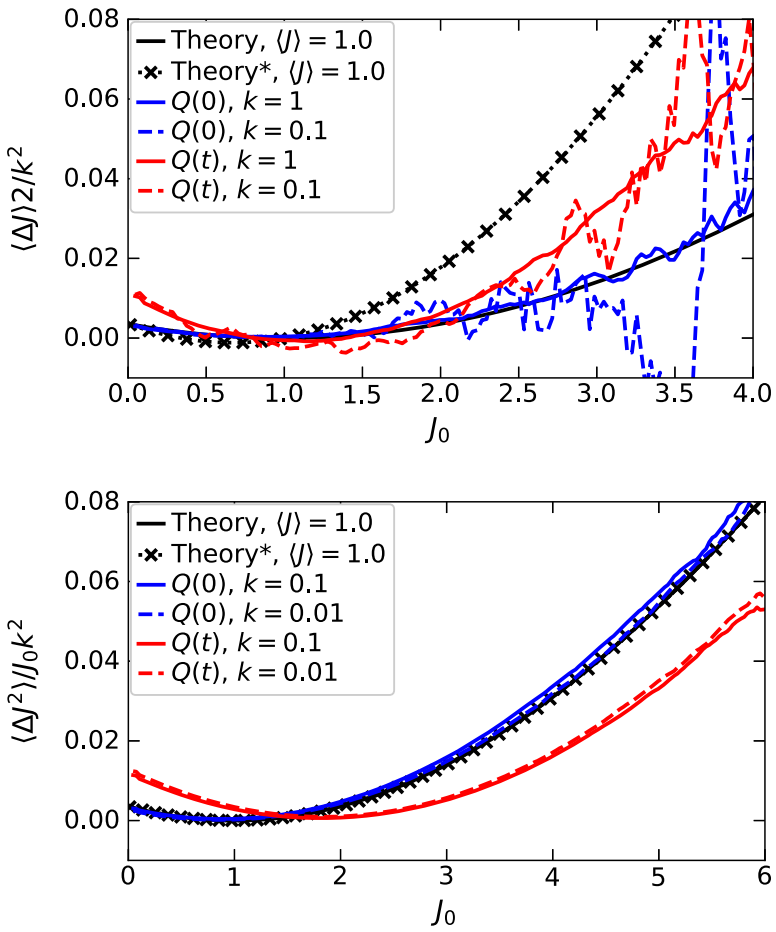

FIG. 3. Change of action after a single kick of amplitude $k$ as a function of the particles' initial action. Macroparticle simulations were run with constant tunes, $Q(0)$, and dynamically evolving tunes, $Q(t)$. They are compared to a theory assuming constant tunes, and a modified theory*, given by Eq. (10) and Eq. (12), respectively. value for $\left\langle\Delta J^{2}\right\rangle$ to first order. The simulated curves for $\langle\Delta J\rangle$ have more statistical noise, as the average is proportional to $k^{2}$, while the spread is proportional to $k \sqrt{J_{0}}$, which is why the curves stop at $J_{0}=4$ and were not calculated for $k<0.1$. The agreement is excellent between Eq. (10) and the simulations with constant tune, denoted by $Q(0)$. The expression in Eq. (10) has thus been verified numerically, using the same assumptions. The agreement is acceptable, for this study, for the simulations run with dynamically evolving tunes, $Q(t)$. The difference appears not to diminish for smaller kicks $k$. The dependence on the dynamical tunes does have an impact. The difference is similar for $\langle\Delta J\rangle$ and $\left\langle\Delta J^{2}\right\rangle$, using the modified theory.

\section{B. Fokker-Planck vs macroparticle simulation}

In the derivation of Eq. (10), it was assumed that the amplitude of the stochastic process was solely dependent on the particle parameters before the kick. That does not represent actual beam dynamics well [11]. The tunes do change during the decoherence process following the kick, which has been accounted for in Eq. (12). Such a process can be modeled by the Fokker-Planck equation in Eq. (18). The distribution then evolves toward a step where $\Delta Q=0$, as seen in Fig. 4. Here, we have studied a 1D toy configuration with $a_{x}=5 \times 10^{-5}, b_{x}=0$, and $g_{x}=$ 0.01 , in the horizontal plane. The time is scaled to hours of operation in the LHC, with a noise of $\sigma_{k}=5 \times 10^{-4}$. The edge develops at $r=\sqrt{2} \approx 1.4$, where $\Delta Q=0$. This is the effect of the diffusion: $\Psi$ increases at $J \lesssim J(\Delta Q=0)$ and at large $J$, and is depleted at $J \gtrsim J(\Delta Q=0)$ and at $J \sim 0$. In other words, $\Psi$ flattens in the two regions separated by $J(\Delta Q=0)=1$.

In simulations with multiple small uncorrelated kicks, the distribution does evolve approximately as dictated by the PDE in Eq. (18), with minor differences [11]. The continuum model does not include the oscillations of the centroid after the kicks, before it has been damped. The amplitude of these oscillations are assumed small,

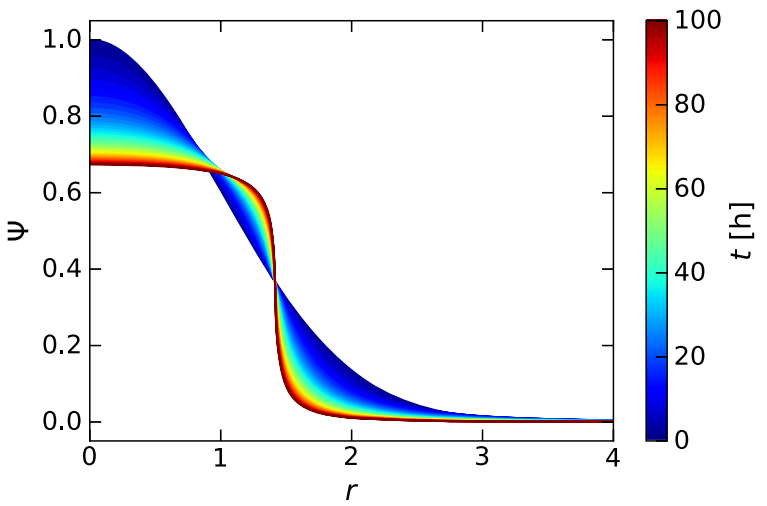

FIG. 4. Distribution evolution with $a_{x}=5 \times 10^{-5}, b_{x}=0$, $g_{x}=0.01$, and noise of $\sigma_{k}=5 \times 10^{-4}$, based on Eq. (18). The time is given by the color scale, going to $100 \mathrm{~h}$. 
compared to the beam size. Nevertheless, it will lead to a nonzero diffusion across the border of zero diffusion coefficient at $\Delta Q=0$. Therefore, even though a clear step can develop in macroparticle simulations, the evolution calculated with Eq. (18) becomes inaccurate as the distribution approaches a hard step. We will abide by this constraint in the following.

The effect we are trying to study, the evolution of the stability diagram due to amplitude dependent transverse diffusion, caused by the combined effect of amplitude dependent detuning and active feedback, is difficult to assess with macroparticle simulations. Relevant parameters for this effect in the LHC are $g \sim 0.1, \Delta Q \sim 10^{-4}$, $\sigma_{k} \sim 10^{-4}, N_{p} \sim 10^{11}$ particles per bunch, $T \sim 10^{7}$ turns. There are multiple numerical obstacles: (i) The process is according to Fig. 2 strongly dependent on the ratio $\Delta Q / g \sim 10^{-3} \ll 1$, and altering it will correspond to a different machine configuration; (ii) The centroid offset depends on the noise and feedback gain as $\left\langle\sqrt{\langle x\rangle^{2}}\right\rangle=\sigma_{k} / \sqrt{2 g} \sim 2 \times 10^{-4} \ll 1$, neglecting the damping from the decoherence. If the centroid offset becomes significantly larger, e.g., if one increases $\sigma_{k}$ to reduce the necessary number of turns in the simulation, the detuning per particle will be averaged over a wider range, leaving the assumption of a weak noise perturbation, and the diffusion becomes closer to uniform; (iii) Due to a limited number of macroparticles, the ideal feedback causes unphysical, numerical stochastic cooling. This effect is weaker for larger $N_{p} / g$ (perhaps with exponents larger than 1); (iv) The rate of change in the distribution is for small $\Delta Q / g$ proportional to $T \sigma_{k}^{2} \Delta Q^{2} / g^{2}$. Bringing it all together, a good simulation will have a large $N_{p} T \cdot \Delta Q^{2} / g^{2} \cdot \sigma_{k}^{2} / g \sim N_{p} T \cdot 4 \times 10^{-14}$. The complexity of the simulations will approximately be proportional to $N_{p} T$, which will have to be large. Experience has shown that $N_{p} T \geq 10^{13}$, with $N_{p} \sim T$, is necessary to get an estimate of this effect, but with further improvement achievable for higher $N_{p} T$. Assuming a perfect simulation, it is also required to have an even higher $N_{p}$ to interpolate the macroparticle bunch to get an agreeable expression for $\partial_{J}(\Psi)$. For numerical calculations of the stability diagram, good statistics up to $r=6$ is necessary.

\section{RESULTS}

We will now present results obtained with PyRADISE, using the Fokker-Planck equation in Eq. (20). First, we will display the change in the distribution from solving the PDE. Then, we will consider the change in the stability diagram that is caused by the change in the distribution. Finally, we will present the change of the effective detuning strength, which will be defined in Sec. IV B, in several scans of the relevant parameters. In all configurations we will keep $a_{x}=a_{y}=a, b_{x}=b_{y}=b$ and $g_{x}=g_{y}=g$, and the subscript will not be included in the following.

\section{A. Distribution and stability evolution in 2D}

Now, we consider a more realistic model for the LHC, with $a=5 \times 10^{-5}, g=0.01$, and $\sigma_{k j}=5 \times 10^{-4}$, as in Fig. 4, but also including the dependence of the detuning in one plane on the action in the other plane, by setting $b=-3.5 \times 10^{-5}$. This noise amplitude is compatible with recent experiments in the LHC [9]. First, we consider a configuration with noise in the horizontal plane only, then, with equal noise in both planes. The number of turns $T$ have been scaled to a time variable $t=T / f_{\text {rev }}$, where $f_{\text {rev }}=11.245 \mathrm{kHz}$ is the revolution frequency in the LHC.

The relative change of the distribution after $12 \mathrm{~h}$, with noise in the horizontal plane only, is displayed in Fig. 5(a). This is the same trend for each value of $J_{y}$, as in Fig. 4. The only difference is that $J_{x}\left(\Delta Q_{x}=0\right)$ now depends on $J_{y}$. Therefore, it is more difficult to see that the projection of the new distribution in the $(x, y)$-plane is not Gaussian, than with $b=0$. The stability in the horizontal plane evolves as in Fig. 5(b), and in the vertical plane as in Fig. 5(c). After $24 \mathrm{~h}$, the horizontal stability diagram is partly inside the black dashed curve, which is the stability diagram of the initial distribution with half the detuning strength. The vertical stability decreases initially slightly at positive $\operatorname{Re} \Delta Q_{\text {coh }}$, but there is no extreme reduction of the stability for any real tune shift. However, at $\operatorname{Re} \Delta Q_{\text {coh }} \approx$ $-0.8 \times 10^{-4}$, the drilling of a hole has begun. This is due to the uncommon appearance of a positive distribution gradient, $\partial \Psi / \partial J_{y}>0$, close to $\Delta Q_{x}=0$.

The relative change of the distribution after $12 \mathrm{~h}$, with equal noise in both transverse planes, is displayed in Fig. 6(a). The evolution is driven by both horizontal and vertical diffusion. Where the horizontal diffusion is zero, the particles only diffuse vertically, leading to a zero distribution gradient, $\partial \Psi / \partial J_{y} \approx 0$. Due to perfect symmetry between the two planes, the stability in both planes evolves as in Fig. 6(b). The evolution of the stability diagram is in this case qualitatively similar to the sum of the evolutions in both planes when there was only horizontal noise. There is a reduction of the stability limit at $\operatorname{Re} \Delta Q_{\text {coh }} \sim 0$, especially for weakly negative real coherent tune shifts, where the most problematic coherent modes in the LHC reside [2]. After $24 \mathrm{~h}$, the stability limit has been reduced to almost that of the initial distribution with half the detuning strength.

\section{B. Relative effective detuning strength}

A stability diagram has been calculated for each distribution $\Psi\left(t=t_{k}\right)$ with the correct detuning coefficients, as described in Sec. II D and presented in Figs. 5, 6. In addition, stability diagrams have been calculated for the initial distribution $\Psi(t=0)$ with scaled detuning coefficients $\left(a_{s}, b_{s}\right)=s \cdot(a, b)$. The relative effective detuning strength at time $t_{k}$ is defined as the largest factor $s$ that corresponds to a stability diagram that is completely inscribed in the stability diagram for distribution $\Psi\left(t=t_{k}\right)$. 


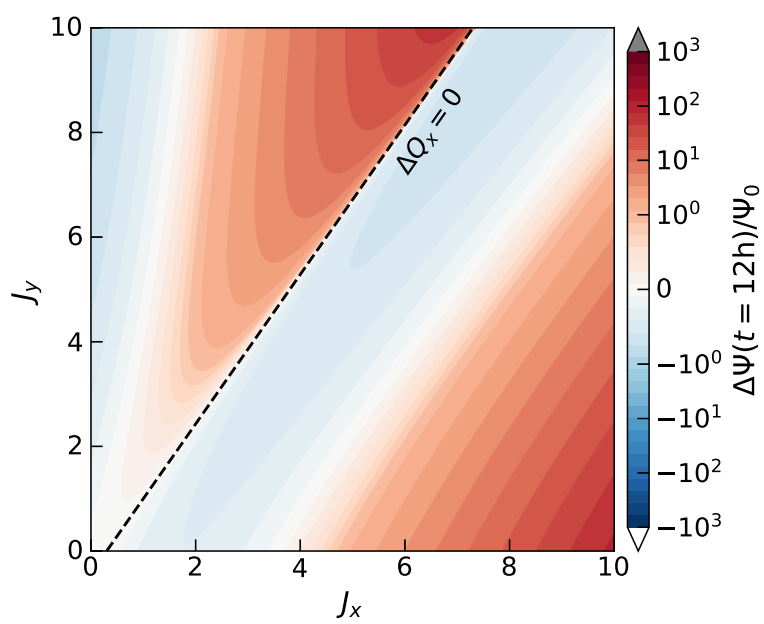

(a)

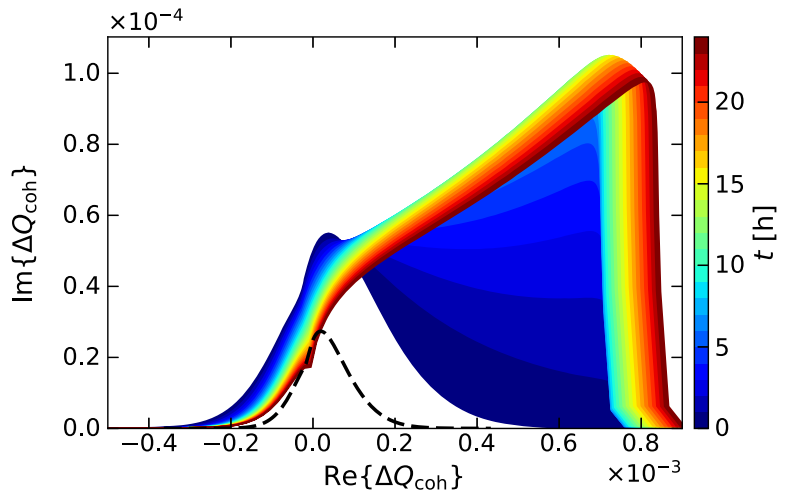

(b)

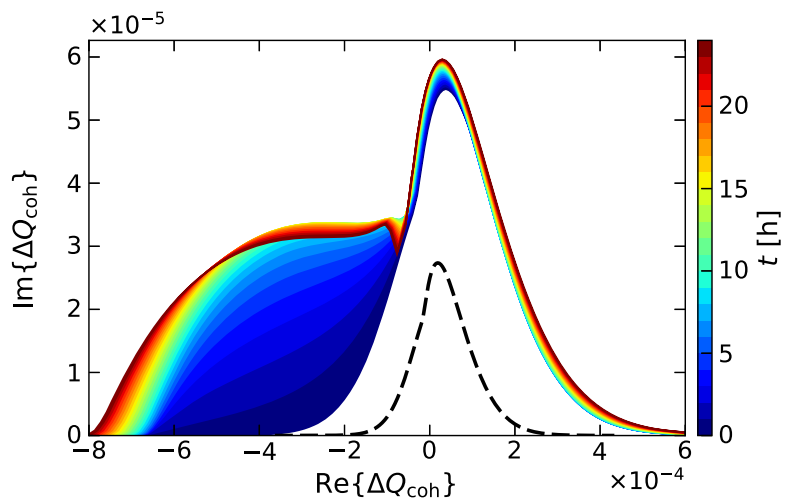

(c)

FIG. 5. Evolution with noise in the horizontal plane only: (a) of the distribution; (b), (c) of the stability diagrams in the horizontal and vertical planes, respectively. The black dashed curves in the stability diagrams correspond to the stability diagrams of the initial distribution with half the detuning strength.

The evolution of the relative effective detuning strengths for the two cases in Sec. IVA are presented in Fig. 7. The decrease of the stability limit is evident, except in the vertical plane when there was no vertical noise. The effective detuning strengths are after $24 \mathrm{~h}$, in these cases, reduced to as low as $42 \%$ of the octupole detuning

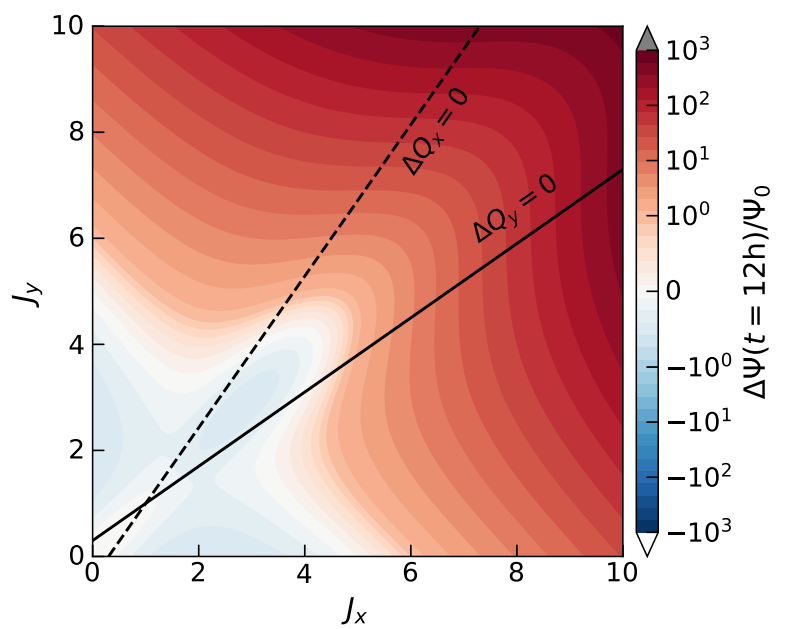

(a)

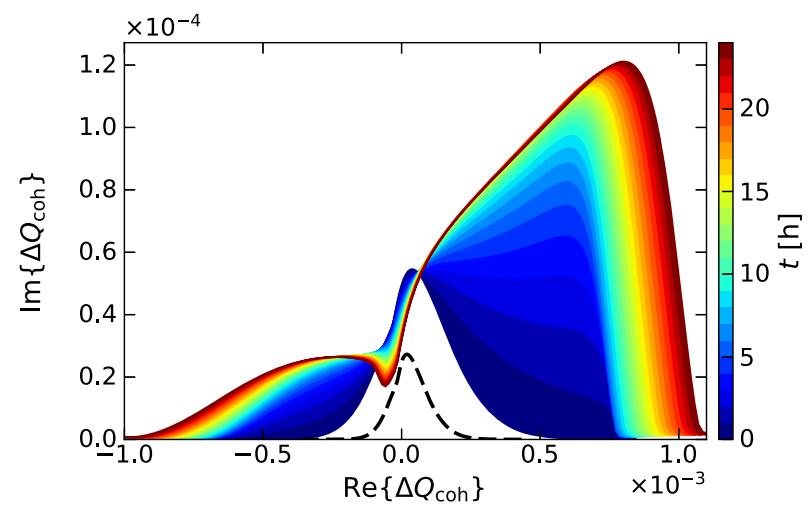

(b)

FIG. 6. Evolution with equal noise and detuning in both planes: (a) of the distribution; (b) of the stability diagram. The stability diagram evolves equally in both planes, due to symmetry. The black dashed curve in the stability diagram corresponds to the stability diagram of the initial distribution with half the detuning strength.

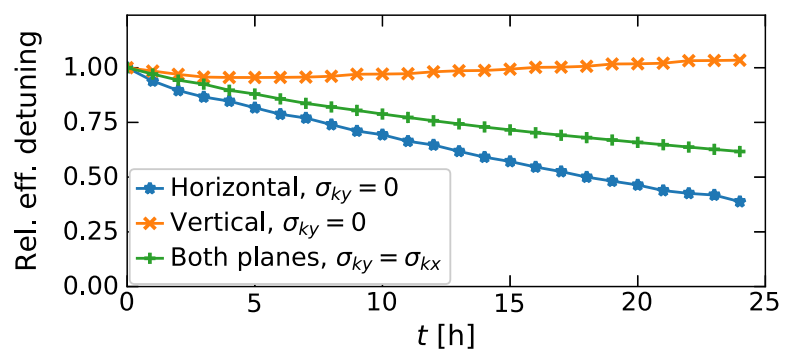

FIG. 7. Evolution of the relative effective detuning strengths, corresponding to the distribution evolutions in Figs. 5 and 6.

one actually has in the machine. That the relative effective detuning strength eventually becomes smaller than 0.5 , is visualized by the red curves in Fig. 5(b) that cross the dashed black line corresponding to a relative detuning strength of $s=0.5$. As seen by the evolution of the stability diagrams in the previous section, the stability does also 


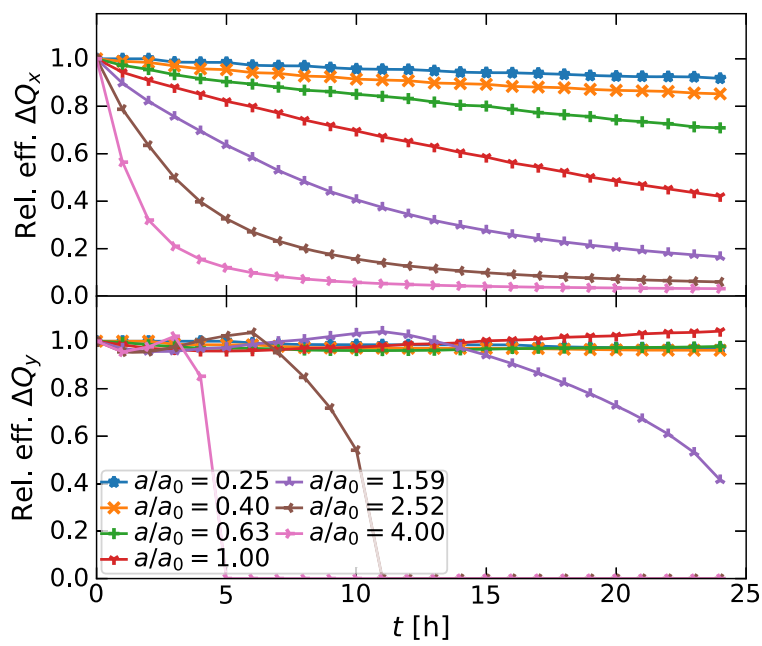

(a)

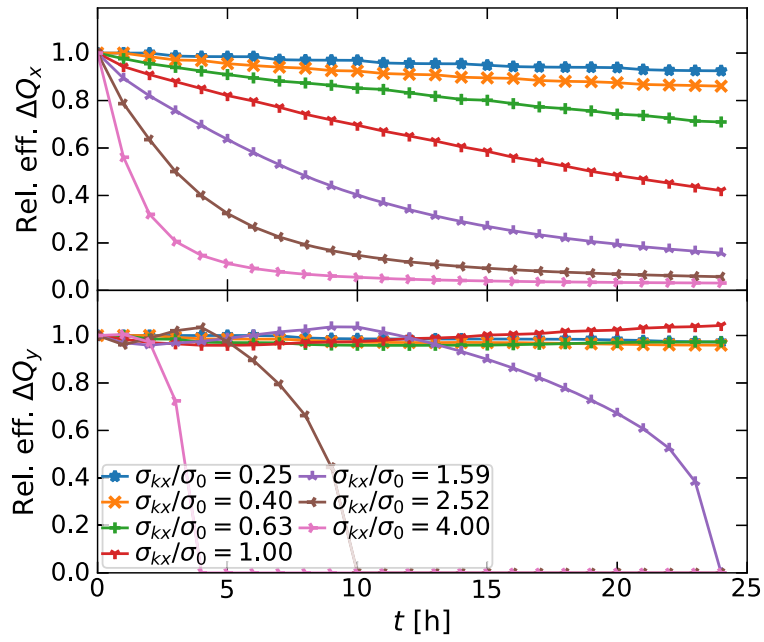

(c)

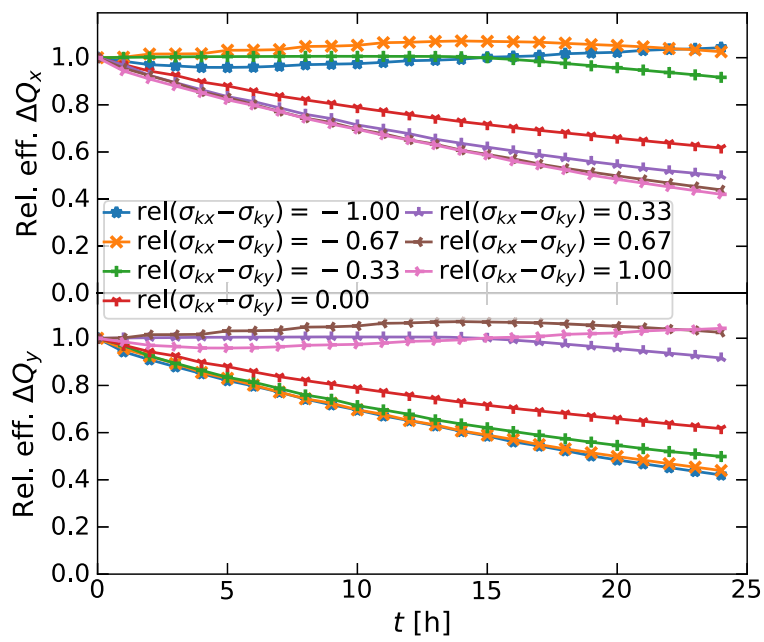

(e)

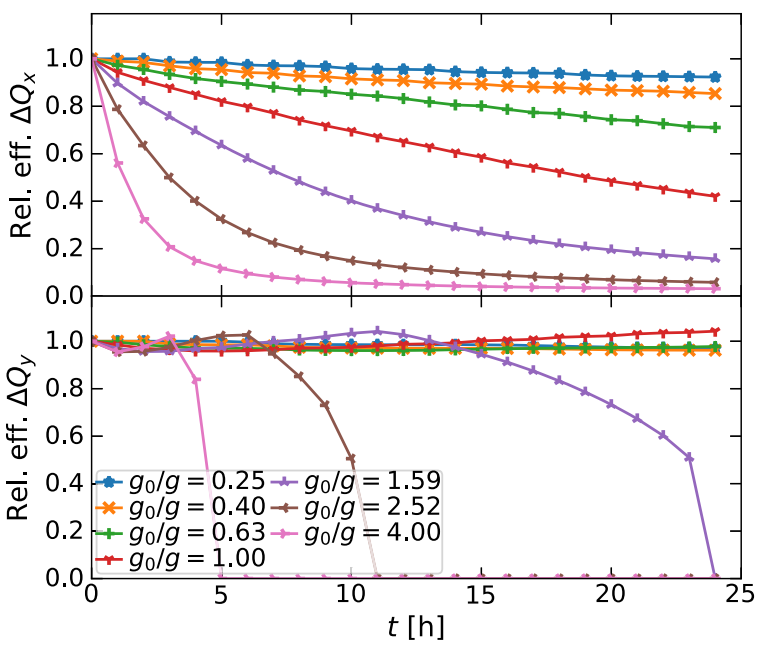

(b)

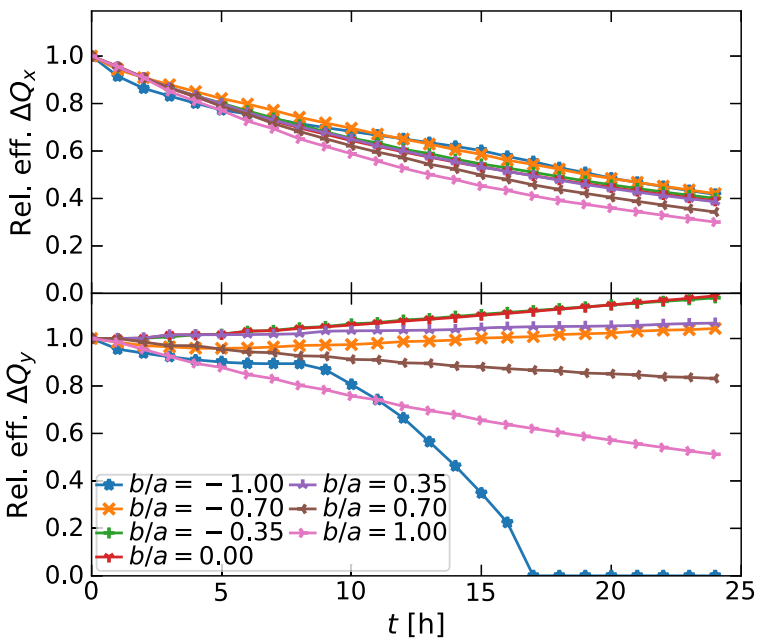

(d)

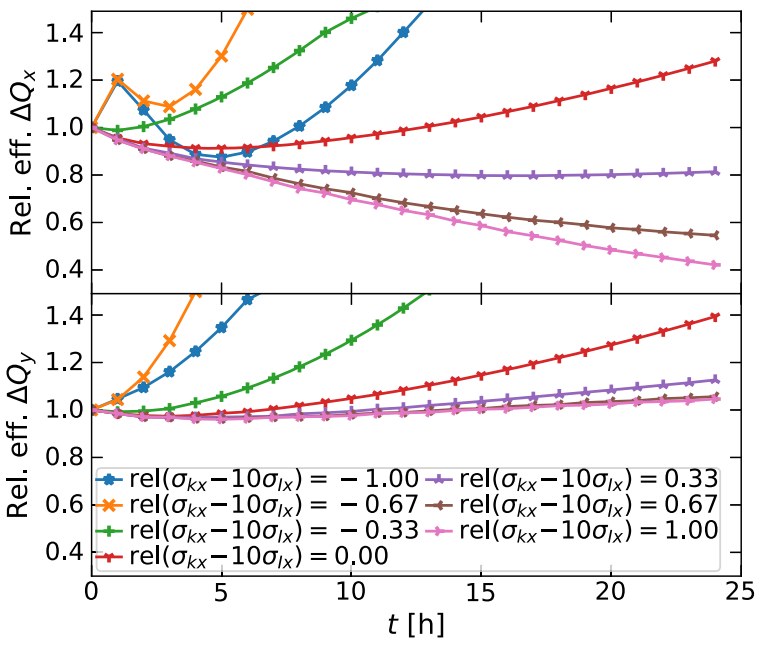

(f)

FIG. 8. Scan of $a, g, \sigma_{k x}, \sigma_{k y}, \sigma_{I x}$, and $b / a$, given by the legends. The relative effective detuning strength is explained in Sec. IV B. The relative difference in (e) and (f) is given by Eq. (27). The configuration in Fig. 5 is the same as $a / a_{0}=1, g_{0} / g=1, \sigma_{k x} / \sigma_{0}=1$, $b / a=-0.7, \operatorname{rel}\left(\sigma_{k x}-\sigma_{k y}\right)=1$, and $\operatorname{rel}\left(\sigma_{k x}-10 \sigma_{I x}\right)=1$, respectively in order from (a) to (f). 
increase for certain values of $\operatorname{Re} \Delta Q_{\text {coh }}$, the relative effective detuning strengths correspond to a worst-case scenario.

\section{Parameter dependence}

It is of both academic and operational interest to investigate how the impact of this mechanism scales with the most important parameters, $a, b, g$, and $\sigma_{k j}$. We have varied these parameters to look for the optimal and worst configurations. The parameters were varied relative to $a_{0}=5 \times 10^{-5}, b_{0}=-0.7 a_{0}, g_{0}=0.01, \sigma_{k x 0}=$ $\sigma_{0}=5 \times 10^{-4}$, and $\sigma_{k y 0}=0$, as in the configuration in Fig. 5.

In the limit $g \gg a$, which is relevant for machine operation, the diffusion coefficients in Eq. (21) mostly depend on the parameter $\sigma_{k j} a / g$. This is confirmed by the scans of $a / a_{0}, g_{0} / g$ and $\sigma_{k x} / \sigma_{0}$, presented in Figs. 8(a), 8(b), and 8(c), respectively. There is a small difference for the largest values of $a / g$, as expected. Note that the largest two values of $\sigma_{k x} a / g$ are included for completeness, but they are not realistic in the LHC as of now. The scans of $a / a_{0}$ and $g_{0} / g$ were repeated while keeping $\sigma_{k x} a$ and $\sigma_{k x} / g$, respectively, constant. In these scans, there were no clear dependences on $a$ or $g$. Note that an increase of $a$ corresponds to an increase of the stable area for $\Delta Q_{\text {coh }}$. Here, we study the relative change of this area. With a larger $a$, there is a larger initial margin for a given mode to go unstable, but the reduction of the margin is also faster, according to these results.

We have also studied the ratio $b / a$. This ratio is typically about -0.7 in the LHC, but it is possible to operate with other ratios, as it mainly depends on the ratio of the transverse $\beta$-functions at the locations of the octupoles [1]. Note that varying $b / a$ will change the shape of the stability diagram. Generally, it is desired to keep $b / a$ negative to generate Landau damping for coherent modes of both positive and negative $\operatorname{Re} \Delta Q_{\text {coh }}$ [5]. The scan of $b / a$ is presented in Fig. 8(d). The evolution of the vertical stability is worst for $b / a \in\{-1,1\}$. In these configurations, the horizontal and vertical isotune curves (curves of equal tunes) in $2 \mathrm{D}$ action space are equal. Therefore, the positive derivative $\partial \Psi / \partial J_{y}$, which is visible in Fig. 5(a), will add up for certain $Q$ in Eq. (1), such that the stability limit on $\operatorname{Im} \Delta Q_{\text {coh }}$ becomes negative. The evolution of the horizontal stability is not strongly dependent on $b / a$.

Next, the vertical noise was increased, and the scan is shown in Fig. 8(e). The noise amplitudes $\sigma_{k x}$ and $\sigma_{k y}$ were varied such that $\max \left(\sigma_{k x}, \sigma_{k y}\right)=\sigma_{0}$, and the relative difference $\operatorname{rel}\left(\sigma_{k x}-\sigma_{k y}\right)$ was scanned, which we have defined as

$$
\operatorname{rel}(\alpha-\beta)=\frac{\alpha-\beta}{\alpha+\beta}, \quad \alpha, \beta \geq 0
$$

Due to an otherwise perfect symmetry, the change of the relative effective horizontal detuning strength for $\operatorname{rel}\left(\sigma_{k x}-\sigma_{k y}\right)=d$ is equal to the relative effective vertical detuning strength for $\operatorname{rel}\left(\sigma_{k x}-\sigma_{k y}\right)=-d$. The relative effective detuning strength in a plane is reduced the fastest with noise in only that plane, and the least with noise in only the other plane, as was already seen in Sec. IVA.

At last, we introduced an incoherent noise amplitude $\sigma_{I j}$, to model in a simplified manner the stochastic process generated by intrabeam scattering [22]. The incoherent noise amplitude corresponds to a uniform diffusion coefficient $D_{I}=\sigma_{I}^{2} / 2 \tau$. Such a uniform diffusion will cause a distribution to become more Gaussian, and will therefore counteract the destabilizing change in the distribution, driven by the coherent noise. A scan with noise in the horizontal plane only is shown in Fig. 8(f). The vertical noise was kept at zero. The horizontal noise amplitudes $\sigma_{k x}$ and $\sigma_{I x}$ were varied to scan $\operatorname{rel}\left(\sigma_{k x}-10 \sigma_{I x}\right)$ from -1 to 1 , keeping $\sigma_{k x}=\sigma_{0}$, except for when the relative difference is -1 , in which case $\sigma_{k x}=0$ and $\sigma_{I x}=\sigma_{0} / 2$. The incoherent noise is beneficial for long-term beam stability. However, after $\sim 4 \mathrm{~h}$, there was a reduction of the stability for $\operatorname{rel}\left(\sigma_{k x}-10 \sigma_{I x}\right) \leq-0.67$. It has been found that this happens because the beam size grows toward the aperture at $J_{\max }$, such that the beam becomes Gaussian with its tails collimated at a certain amplitude [5,23]. With the strongest incoherent noise, the beam size was doubled after $142 \mathrm{~min}$, and $83 \%$ of the bunch intensity was lost after $24 \mathrm{~h}$. For strong incoherent noise, the nonuniform diffusion due to the coherent noise is actually beneficial for long-term
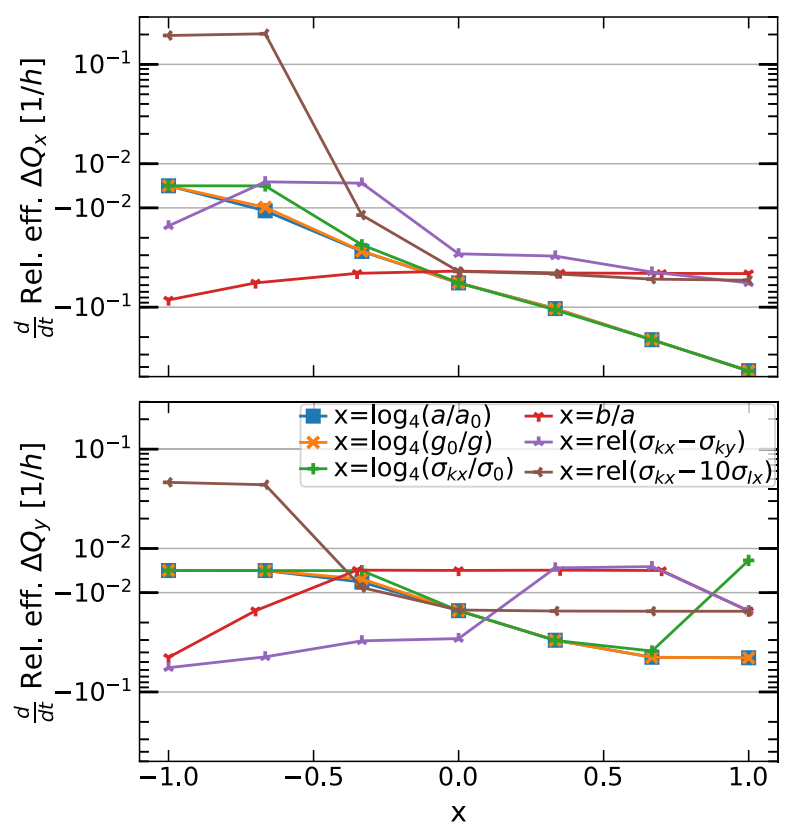

FIG. 9. The initial reduction of the relative effective detuning strength, from the scans that are given in more detail in Fig. 8. The value corresponds to the change during the first hour, after starting with a Gaussian transverse distribution. The vertical scale is linear on the interval $\left[-10^{-2}, 10^{-2}\right]$, and logarithmic otherwise. 
stability. The increase of the relative effective detuning strength beyond 1.5 is not of interest.

The initial change of the relative effective detuning strength per hour, for all the parameter scans, is presented in Fig. 9. The reduction is faster due to noise in the same plane, with $b / a=-1$, and with no incoherent noise. To a certain extent, it does not matter how one changes $\sigma_{k x} a / g$. Except at large values of this parameter, there is never a reduction of the relative effective detuning strength faster than $10 \% / \mathrm{h}$. Hence, this effect cannot reduce the stability substantially within a latency time of $\lesssim 30 \mathrm{~min}$, which has been observed in the LHC [9].

\section{CONCLUSION}

The intrabunch motion of particles have in this study been considered in detail. It has been found that due to the combined mechanism of linear detuning and transverse feedback, the change of action after a kick depends on the tune of the individual particle, relative to the average tune of the distribution. By considering the change of action after an initial kick, due to a noise source, as a stochastic process, the Fokker-Planck equation has been derived from a master equation. The resulting diffusion coefficient is 0 for particles with tune equal to the average tune of the bunch, and grows quadratically with the tune from there. While incoherent noise, such as intrabeam scattering, causes the distribution to tend to a Gaussian, the coherent noise causes the distribution to tend to a step function.

The change in the distribution from a Gaussian toward a step, changes the stability diagram as well. The change of the stability limit has been studied through the change of the relative effective detuning, a worst-case measure. The relative effective detuning strength in the horizontal plane decreased faster, and thus approached an instability faster, with: (i) larger absolute values of the detuning coefficients $a$ and $b\left(\Delta Q_{x}=a J_{x}+b J_{y}\right)$; (ii) smaller damper gain $g$; (iii) stronger coherent noise in the same plane $\sigma_{k x}$; (iv) coherent noise in only the opposite plane, combined with $b / a=-1$; (v) larger ratio of the coherent noise amplitude in the same plane to that in the other plane, $\sigma_{k x} / \sigma_{k y}$; (vi) larger ratio of the coherent noise to the incoherent noise $\sigma_{k x} / \sigma_{I x}$. The relative effective detuning strength was reduced by up to $58 \%$ in a realistic configuration, over a time period of $24 \mathrm{~h}$. It was not found a reduction of the relative effective detuning strength higher than $10 \% / \mathrm{h}$ with $\mathrm{LHC}$ relevant parameters. Hence, this mechanism does not explain the latencies measured in the LHC.

The mechanism considered here is not critical for beam stability in the LHC. Previous considerations [3] and recent developments $[24,25]$ imply that a more critical mechanism is a diffusion that is peaked and narrow in frequency space, driven by wakefields, requiring a nonlinear treatment, or by a narrow band noise source, centered inside the beam spectrum. In the future, it will be of interest to include that mechanism in the formalism presented here.

\section{ACKNOWLEDGMENTS}

The authors would like to thank both E. Métral and G. Arduini for proofreading this manuscript.

\section{APPENDIX A: CHANGE OF ACTION}

In this Appendix, we derive the expression for the change of action in Eq. (10). To do so, we refer to the centroid of the bunch as

$$
z=\langle x\rangle+\mathrm{i}\langle p\rangle .
$$

The tune of the centroid is denoted $Q_{c}$, and its transverse offset will be reduced by the transverse feedback toward the design trajectory by a factor called the gain $g$. Assuming a perfect, immediate feedback, the evolution of the centroid from one turn to the next is given by

$$
z_{1}=z_{0} \mathrm{e}^{-\mathrm{i} 2 \pi Q_{c}}\left(1-\frac{g}{2}\right)
$$

The initial centroid offset, $z_{0}=\mathrm{i} k$, will after $n$ turns be

$z_{n}=z_{0} \mathrm{e}^{-\mathrm{i} 2 \pi Q_{c} n}\left(1-\frac{g}{2}\right)^{n} \stackrel{n \rightarrow \infty}{\rightarrow} z_{0} \mathrm{e}^{-\mathrm{i} 2 \pi Q_{c} n} \mathrm{e}^{-\frac{g}{2} n}$,

with a damping time of $\tau=2 / g$ turns. It is assumed that the reduction of the centroid amplitude due to the tune spread is negligible compared to that of the transverse feedback.

The position of an individual particle, with a constant tune offset $\Delta Q$ from the centroid, is referred to as

$$
y=x+\mathrm{i} p .
$$

After many turns, when the centroid tends to the origin in the limit of $n g \gg 1$, the position will become

$$
\begin{aligned}
y_{n} & =\mathrm{e}^{-\mathrm{i} 2 \pi \sum_{j=0}^{n-1}\left(Q_{c}+\Delta Q_{j}\right)}\left(y_{0}-z_{0} \frac{g}{2} \sum_{j=0}^{n-1}\left(1-\frac{g}{2}\right)^{j} \mathrm{e}^{\mathrm{i} 2 \pi \sum_{l=0}^{j-1} \Delta Q_{l}}\right) \\
& =\mathrm{e}^{-\mathrm{i} 2 \pi\left(Q_{c}+\Delta Q\right) n}\left(y_{0}-z_{0} \frac{g}{\left.21-\left(1-\frac{g}{2}\right) \mathrm{e}^{\mathrm{i} 2 \pi \Delta Q}\right),}\right.
\end{aligned}
$$

where $y_{0}$ is the position of the particle just after the kick $z_{0}$. In going from line 1 to line 2, one has assumed that $Q_{c}$ and $\Delta Q$ are constant during the process, and one has taken the sum of the geometric series. Assuming the kicks are small, this expression can easily be extended to include more kicks as $z_{0} \rightarrow \sum_{j=0}^{m} \mathrm{i} k_{j} \mathrm{e}^{\mathrm{i} 2 \pi Q j}$. 
Equation (A5) can be rewritten as

$$
y_{n}=\mathrm{e}^{-\mathrm{i} 2 \pi\left(Q_{c}+\Delta Q\right) n}\left(r_{0}+z_{0} \frac{\left(1-\frac{g}{2}\right)\left(1-\mathrm{e}^{\mathrm{i} 2 \pi \Delta Q}\right)}{1-\left(1-\frac{g}{2}\right) \mathrm{e}^{\mathrm{i} 2 \pi \Delta Q}}\right),
$$

where $r_{0}$ is the position of the particle prior to the kick, compared to $y_{0}=r_{0}+z_{0}$, which is the position immediately after the kick.

It follows from Eq. (3) that

$$
2 J_{n}=x^{2}+p^{2}=y^{*} y=|y|^{2},
$$

where the $*$ signifies that it is the complex conjugate. Filling in, remembering that $r_{0}$ and $z_{0}$ are complex numbers,

$$
\begin{aligned}
2 J & =\mathrm{e}^{\mathrm{i} 2 \pi\left(Q_{c}+\Delta Q\right) n}\left(r_{0}^{*}+z_{0}^{*} \frac{\left(1-\frac{g}{2}\right)\left(1-\mathrm{e}^{-\mathrm{i} 2 \pi \Delta Q}\right)}{1-\left(1-\frac{g}{2}\right) \mathrm{e}^{-\mathrm{i} 2 \pi \Delta Q}}\right) \times \mathrm{e}^{-\mathrm{i} 2 \pi\left(Q_{c}+\Delta Q\right) n}\left(r_{0}+z_{0} \frac{\left(1-\frac{g}{2}\right)\left(1-\mathrm{e}^{\mathrm{i} 2 \pi \Delta Q}\right)}{1-\left(1-\frac{g}{2}\right) \mathrm{e}^{\mathrm{i} 2 \pi \Delta Q}}\right) \\
& =\left|r_{0}\right|^{2}+\left|z_{0}\right|^{2} \frac{\left(1-\frac{g}{2}\right)^{2}\left(1-\mathrm{e}^{-\mathrm{i} 2 \pi \Delta Q}\right)\left(1-\mathrm{e}^{\mathrm{i} 2 \pi \Delta Q}\right)}{1+\left(1-\frac{g}{2}\right)\left(1-\frac{g}{2}-\mathrm{e}^{-\mathrm{i} 2 \pi \Delta Q}-\mathrm{e}^{\mathrm{i} 2 \pi \Delta Q}\right)}+\left(r_{0}^{*} z_{0} \frac{\left(1-\frac{g}{2}\right)\left(1-\mathrm{e}^{\mathrm{i} 2 \pi \Delta Q}\right)}{1-\left(1-\frac{g}{2}\right) \mathrm{e}^{\mathrm{i} 2 \pi \Delta Q}}+\text { c.c. }\right) \\
& =2 J_{0}+\left|z_{0}\right|^{2} f_{1}+\left(r_{0}^{*} z_{0} f_{2}+\text { c.c. }\right),
\end{aligned}
$$

where the factors depending on $g$ and $\Delta Q$ have been renamed $f_{1}$ and $f_{2}$.

We will now make use of the well-known expressions

$$
\begin{gathered}
2 \cos (x)=\mathrm{e}^{\mathrm{i} x}+\mathrm{e}^{-\mathrm{i} x}, \\
2 \mathrm{i} \sin (x)=\mathrm{e}^{\mathrm{i} x}-\mathrm{e}^{-\mathrm{i} x}, \\
\cos (2 x)=1-2 \sin ^{2}(x), \\
\mathrm{e}^{\mathrm{i} x}=\cos (x)+\mathrm{i} \sin (x) .
\end{gathered}
$$

By insertion for $f_{1}$ one finds that

$$
f_{1}=\frac{\left(1-\frac{g}{2}\right)^{2}\left(-4 \mathrm{i}^{2}\right) \sin ^{2}(\pi \Delta Q)}{1+\left(1-\frac{g}{2}\right)\left(1-\frac{g}{2}-2 \cos (2 \pi \Delta Q)\right)}=\frac{\left(1-\frac{g}{2}\right)^{2} 4 \sin ^{2}(\pi \Delta Q)}{\left(\frac{g}{2}\right)^{2}+\left(1-\frac{g}{2}\right) 4 \sin ^{2}(\pi \Delta Q)} .
$$

For $f_{2}$, we first multiply with and divide by the complex conjugate of the denominator to get

$$
\begin{aligned}
f_{2} & =\frac{\left(1-\frac{g}{2}\right)\left(1-\mathrm{e}^{\mathrm{i} 2 \pi \Delta Q}\right)}{1-\left(1-\frac{g}{2}\right) \mathrm{e}^{\mathrm{i} 2 \pi \Delta Q}} \cdot \frac{1-\left(1-\frac{g}{2}\right) \mathrm{e}^{-\mathrm{i} 2 \pi \Delta Q}}{1-\left(1-\frac{g}{2}\right) \mathrm{e}^{-\mathrm{i} 2 \pi \Delta Q}}=\left(1-\frac{g}{2}\right) \frac{\left[2-\frac{g}{2}-2 \cos (2 \pi \Delta Q)+\left(\frac{g}{2}\right) \mathrm{e}^{-\mathrm{i} 2 \pi \Delta Q}\right]}{1+\left(1-\frac{g}{2}\right)^{2}-\left(1-\frac{g}{2}\right) 2 \cos (2 \pi \Delta Q)} \\
& =\left(1-\frac{g}{2}\right) \frac{(4-g) \sin ^{2}(\pi \Delta Q)-\mathrm{i}\left(\frac{g}{2}\right) \sin (2 \pi \Delta Q)}{\left(\frac{g}{2}\right)^{2}+\left(1-\frac{g}{2}\right) 4 \sin ^{2}(\pi \Delta Q)} .
\end{aligned}
$$

To get an expression for the last parenthesis in Eq. (A8), we note that for a complex number $c, c+c^{*}=2 \operatorname{Re} c$, and that $r_{0}^{*} z_{0}=p_{0} k+\mathrm{i} x_{0} k$. Dividing by 2 , and setting $\Delta J=J_{n}-J_{0}$, gives

$$
\Delta J=\frac{k^{2}}{2} \frac{\left(1-\frac{g}{2}\right)^{2} 4 \sin ^{2}(\pi \Delta Q)}{\left(\frac{g}{2}\right)^{2}+\left(1-\frac{g}{2}\right) 4 \sin ^{2}(\pi \Delta Q)}+k\left(1-\frac{g}{2}\right) \frac{x_{0}\left(\frac{g}{2}\right) \sin (2 \pi \Delta Q)+p_{0}(4-g) \sin ^{2}(\pi \Delta Q)}{\left(\frac{g}{2}\right)^{2}+\left(1-\frac{g}{2}\right) 4 \sin ^{2}(\pi \Delta Q)},
$$

which is identical to Eq. (8) when Taylor expanding the sine functions to first order in the limit $\Delta Q \ll 1$, and using the expressions for $x_{0}$ and $p_{0}$ in Eq. (2). 


\section{APPENDIX B: DERIVING FOKKER-PLANCK}

In this Appendix we aim at deriving the Fokker-Planck equation described by Eqs. (14)-(15). We begin by finding the master equation, similarly to what was done in [15], by taking a convolution of the distribution before the change, $\Psi(t)$, with the probability distribution of the change, $\varphi(\Delta)$,

$\Psi(J, t+\tau)=\int_{-\infty}^{\infty} \Psi(J-\Delta, t) \varphi(\Delta ; J-\Delta, \Psi) \mathrm{d} \Delta$,

where $\Delta$ represents the change of action. The action will only change in the plane of the kick.

The next step is to Taylor expand the integrand around $J$, to get to the third order

$$
\begin{aligned}
\Psi(J, t+\tau)= & \int_{-\infty}^{\infty}\{\Psi(J) \varphi(\Delta ; J) \\
& -\Delta \cdot \partial_{J}[\Psi(J) \varphi(\Delta ; J)] \\
& \left.+\frac{\Delta^{2}}{2} \cdot \partial_{J}^{2}[\Psi(J) \varphi(\Delta ; J)]\right\} \mathrm{d} \Delta,
\end{aligned}
$$

where we have omitted the dependence on $\Psi$ and $t$ for readability. The distribution $\Psi(J, t)$ and the derivative $\partial_{J}$. does not depend on $\Delta$, and can be taken outside the integral, while the change magnitude $\Delta$ is a variable that does not depend on $J$, and can be taken inside the partial differentiation. The integral of the first term is the normalization integral, which is 1 , and we get

$$
\begin{aligned}
\Psi(J, t+\tau)-\Psi(J, t)= & -\partial_{J}\left[\Psi(J) \int_{-\infty}^{\infty} \Delta \varphi(\Delta ; J) \mathrm{d} \Delta\right] \\
& +\partial_{J}^{2}\left[\Psi(J) \int_{-\infty}^{\infty} \frac{\Delta^{2}}{2} \varphi(\Delta ; J) \mathrm{d} \Delta\right] .
\end{aligned}
$$

Divide by the time $\tau$ of this process to get a time derivative on the left, and we arrive at the Fokker-Planck equation [17]

$$
\partial_{t} \Psi=-\partial_{J}(U \Psi)+\partial_{J}^{2}(D \Psi),
$$

with drift and diffusion coefficients given respectively by

$$
\begin{aligned}
& U(J, \Psi)=\int_{-\infty}^{\infty} \frac{\Delta}{\tau} \varphi(\Delta ; J, \Psi) \mathrm{d} \Delta, \\
& D(J, \Psi)=\int_{-\infty}^{\infty} \frac{\Delta^{2}}{2 \tau} \varphi(\Delta ; J, \Psi) \mathrm{d} \Delta .
\end{aligned}
$$

$D$ is normalized by $J$ in Eq. (14) for later convenience.

[1] J. S. Berg and F. Ruggiero, Landau damping with twodimensional betatron tune spread, CERN Report No. CERN-SL-AP-96-071-AP, 1996.
[2] N. Mounet, The LHC transverse coupled-bunch instability, Ph.D. thesis, Inst. of Physics, École polytechnique fédérale de Lausanne, Lausanne, Switzerland, 2012, CERNTHESIS-2012-055.

[3] X. Buffat, Transverse beams stability studies at the Large Hadron Collider, Ph.D. thesis, Inst. of Physics, École polytechnique fédérale de Lausanne, Lausanne, Switzerland, 2015, CERN-THESIS-2014-246.

[4] A. G. Ruggiero and G. V. Vaccaro, Solution of the dispersion relation for longitudinal stability of an intense coasting beam in a circular accelerator (Application to the ISR), CERN Report No. CERN-ISR-TH-68-33, 1968.

[5] E. Métral and A. Verdier, Stability diagram for Landau damping with a beam collimated at an arbitrary number of sigmas, CERN Report No. CERN-AB-2004-019-ABP, 2004.

[6] C. Tambasco, J. Barranco, X. Buffat, and T. Pieloni, Transverse beam stability and Landau damping in hadron colliders, presented at FCC week 2018, Amsterdam, Netherlands, May 2018, https://indico.ihep.ac.cn/event/ 6618/session/13/contribution/145.

[7] G. Dôme, Diffusion due to RF noise, in Proceedings of CERN Accelerator School (Oxford, England, 1985) CERN Report No. 87-03, 1987.

[8] A. Bazzani and L. Beccaceci, Diffusion in Hamiltonian systems driven by harmonic noise, J. Phys. A 31, 5843 (1998).

[9] S. V. Furuseth, D. Amorim, S. A. Antipov, X. Buffat, N. Mounet, E. Métral, T. Pieloni, B. Salvant, and C. Tambasco, Instability latency in the LHC, in Proceedings of 10th International Particle Accelerator Conference, Melbourne, Australia (JaCOW, Melbourne, Australia, 2019), pp. 32043207, https://doi.org/10.18429/JACoW-IPAC2019-WEPT S044.

[10] S. V. Furuseth, X. Buffat, E. Métral, D. Valuch, B. Salvant, D. Amorim, N. Mounet, M. Söderén, S. A. Antipov, T. Pieloni, and C. Tambasco, MD3288: Instability latency with controlled noise, CERN Report No. CERN-ACCNOTE-2019-0011, 2019.

[11] S. V. Furuseth and X. Buffat, Change of beam distribution due to decoherence in the presence of transverse feedback, J. Phys. Conf. Ser. 1350, 012118 (2019).

[12] X. Buffat, W. Herr, N. Mounet, T. Pieloni, and S. White, Stability diagrams of colliding beams in the Large Hadron Collider, Phys. Rev. Accel. Beams 17, 111002 (2014).

[13] A. Wolski, Beam Dynamics in High Energy Particle Accelerators (Imperial College Press, London, England, 2014).

[14] V. A. Lebedev, Emittance growth due to noise and its suppression with the feedback system in large hadron colliders, in AIP Conference Proceedings Accelerator physics at the Superconducting Super Collider, Dallas, Texas, USA (AIP Publishing, Dallas, Texas, 1995), pp. 396-423, https://doi.org/10.1063/1.47298.

[15] A. Einstein, Über die von der molekularkinetischen Theorie der Wärme geforderte Bewegung von in ruhenden Flüssigkeiten suspendierten Teilchen, Ann. Phys. (Berlin) 322, 549 (1905). 
[16] F. Sattin, Fick's law and Fokker-Planck equation in inhomogeneous environments, Phys. Rev. Lett. A 372, 3941 (2008).

[17] H. Risken and T. Frank, The Fokker-Planck Equation: Methods of Solution and Applications (Springer-Verlag, Berlin, Heidelberg, New York, 1996).

[18] S. V. Furuseth and X. Buffat, Modeling of nonlinear effects due to head-on beam-beam interactions, Phys. Rev. Accel. Beams 21, 081002 (2018).

[19] scipy.integrate.solve_ivp, https://docs.scipy.org/doc/scipy/ reference/generated/scipy.integrate.solve_ivp.html, accessed 2019-10-28.

[20] J. Gareyte, J. P. Koutchouk, and F. Ruggiero, Landau damping, dynamic aperture and octupoles in LHC, CERN Report No. CERN-LHC-PROJECT-REPORT-091, 1997.

[21] S. Fartoukh, Achromatic telescopic squeezing scheme and its application to the LHC and its luminosity upgrade, Phys. Rev. Accel. Beams 16, 111002 (2013).
[22] A. Piwinski, Intra-beam-scattering, in Proceedings of the 9th International Conference on High Energy Accelerators, Stanford, CA, 1974, p. 405, https://doi.org/10.5170/ CERN-1992-001.226.

[23] G. Arduini, Potential performance reach for the HL-LHC in case of a depleted beam halo, in Review of the Needs for a Hollow e-lens for the HL-LHC (CERN, Geneva Switzerland, 2016), https://indico.cern.ch/event/567839/contributions/22 95334/.

[24] S. V. Furuseth and X. Buffat, Noise and possible loss of Landau damping-noise excited wakefields, in ICFA mini workshop on Mitigation of Coherent Beam Instabilities in particle accelerators, Zermatt, Switzerland, 2019, https:// indico.cern.ch/event/775147/contributions/3366452/.

[25] V. Lebedev, Transverse dampers with ultimate gain for suppression of instabilities in Large Hadron Colliders, in ICFA mini workshop on Mitigation of Coherent Beam Instabilities in particle accelerators, Zermatt, Switzerland, 2019, https://indico.cern.ch/event/775147/contributions/336 $6412 /$. 